\title{
RX J0806.3+1527: A double degenerate binary with the shortest known orbital period (321s) ${ }^{\star}$
}

\author{
G. L. Israel ${ }^{1, \star \star}$, W. Hummel ${ }^{2}$, S. Covino ${ }^{3}$, S. Campana ${ }^{3, \star \star}$, I. Appenzeller ${ }^{4}$, W. Gässler ${ }^{5,6}$, \\ K.-H. Mantel ${ }^{5}$, G. Marconi ${ }^{7,1}$, C. W. Mauche ${ }^{8}$, U. Munari ${ }^{9}$, I. Negueruela ${ }^{10}$, H. Nicklas ${ }^{11}$, \\ G. Rupprecht ${ }^{2}$, R. L. Smart ${ }^{12}$, O. Stahl ${ }^{4}$, and L. Stella ${ }^{1, \star \star}$
}

1 INAF - Osservatorio Astronomico di Roma, V. Frascati 33, 00040 Monteporzio Catone, Italy

2 European Southern Observatory, Karl-Schwarzschildstr. 2, 85748 Garching, Germany

3 INAF - Osservatorio Astronomico di Brera, via Bianchi 46, 23807 Merate, Italy

4 Landessternwarte Heidelberg, Königstuhl 12, 69117 Heidelberg, Germany

5 Institut für Physik, Universitäts-Sternwarte München, Scheinerstr. 1, 81673 München, Germany

${ }^{6}$ Subaru Telescope, NAOJ, 650 North A'ohoku Place, Hilo, Hawaii, HI 96720, USA

7 European Southern Observatory, Casilla 19001, Santiago, Chile

8 Lawrence Livermore National Laboratory, L-43, 7000 East Avenue, Livermore, CA 94550, USA

9 INAF - Osservatorio Astronomico di Padova, Sede di Asiago, 36012 Asiago, Italy

10 Observatoire Astronomique de Strasbourg, 11 rue de l'Université, 67000 Strasbourg, France

11 Universitätssternwarte Göttingen, Geismarlandstr. 11, 37083 Göttingen, Germany

12 INAF - Osservatorio Astronomico di Torino, Strada Osservatorio 20, 10025, Pino Torinese (To), Italy

Received 12 February 2002 / Accepted 1 March 2002

\begin{abstract}
We carried out optical observations of the field of the X-ray pulsator RX J0806.3+1527. A blue $V=$ 21.1 star was found to be the only object consistent with the X-ray position. VLT FORS spectra revealed a blue continuum with no intrinsic absorption lines. Broad $\left(v \sim 1500 \mathrm{~km} \mathrm{~s}^{-1}\right)$, low equivalent width $(\sim-1 \div-6 \AA)$ emission lines from the HeII Pickering series were clearly detected. $B, V$ and $R$ time-resolved photometry revealed the presence of $\sim 15 \%$ pulsations at the $\sim 321 \mathrm{~s} \mathrm{X}$-ray period, confirming the identification. These findings, together with the period stability and absence of any additional modulation in the $1 \mathrm{~min}-5 \mathrm{hr}$ period range, argue in favour of the orbital interpretation of the $321 \mathrm{~s}$ pulsations. The most likely scenario is thus that RX J0806.3+1527 is a double degenerate system of the AM CVn class. This would make RX J0806.3+1527 the shortest orbital period binary currently known and one of the best candidates for gravitational wave detection.
\end{abstract}

Key words. stars: individual: - RX J0806.3+1527, 1BMW J080622.8+152732 - binaries: close stars: white dwarfs - stars: emission-line - X-rays: stars

\section{Introduction}

During a systematic search for periodic signals in a sample of $\sim 4000$ ROSAT HRI $(0.1-2.4 \mathrm{keV})$ light curves we discovered $321.25 \mathrm{~s}$ pulsations in the $\mathrm{X}$-ray flux of RX J0806.3+1527 (Israel et al. 1999, thereafter I99). Based on the large pulsed fraction $(\sim 100 \%)$, relatively low $0.5-2.0 \mathrm{keV}$ flux $\left(3.0-5.0 \times 10^{-12} \mathrm{erg} \mathrm{cm}^{-2} \mathrm{~s}^{-1}\right)$, modest distance (edge of the Galaxy is at $\leq 1 \mathrm{kpc}$ in the direction of the source) and presence of a faint $(B=20.7)$ blue object in the Digitized Sky Survey 1".5 away from the $\mathrm{X}$-ray position, the source was tentatively classified as a cataclysmic variable of the intermediate polar class (I99).

\footnotetext{
Send offprint requests to: G. L. Israel,

e-mail: gianluca@mporzio.astro.it

* Based on observations carried out at ESO VLT (60.A-9203 and 66.D-0675) and La Silla (64.H-0604).

** Affiliated to I.C.R.A.
}

A similar classification was also suggested by Beuermann et al. (1999; see also Burwitz \& Reinsch 2001) based on the source X-ray colours in the ROSAT all sky survey (RASS).

In this letter we report the results of optical campaigns carried out in 1999-2001, which confirm unambiguously the identification of the optical counterpart of RX J0806.3+1527 suggested by I99 and provide evidence that RX J0806.3+1527 is a double white dwarf interacting binary with the shortest known orbital period.

\section{Observations}

Table 1 gives the journal of observations. Several images in $U, B, V, R$ and $I$ Bessel filters were taken together with three different $\mathrm{H} \alpha$ interference filters $(\Delta \lambda=65 \AA$; obs. A-E). Standard procedures were used for bias subtraction and flat-field correction. Figure 1 shows the 
Table 1. Optical observations carried out in 1999-2001 for RX J0806.3+1527.

\begin{tabular}{|c|c|c|c|c|c|}
\hline $\begin{array}{c}\text { Obs. } \\
\#\end{array}$ & Telescope \& Instrument & Date & $\begin{array}{l}\text { Exp. } \\
\text { (s) }\end{array}$ & $\begin{array}{l}\text { Range } \\
(\text { Band } / \AA)\end{array}$ & Comments \\
\hline $\mathrm{A}$ & $8.2 \mathrm{~m} \mathrm{VLT-UT2} \mathrm{FORS2}$ & 1999/Nov./12 & 300 & $B V R I \mathrm{H}_{\alpha} \mathrm{H}_{\alpha \mathrm{cont}}$ & \\
\hline $\mathrm{B}$ & $"$ & 1999/Nov./16-19 & 10500 & $3600-6000$ & $6 \AA$ res.; grism $600 \mathrm{~B}$; slit $1^{\prime \prime}$ \\
\hline $\mathrm{C}$ & $"$ & 1999/Nov./18 & $600 \div 300$ & $U B V R I$ & \\
\hline $\mathrm{D}$ & $"$ & 2000/Feb./05 & 2700 & $3600-9000$ & $30 \AA$ res.; grism $150 \mathrm{I}$; slit $1^{\prime \prime}$ \\
\hline $\mathrm{E}$ & $3.6 \mathrm{~m} \mathrm{ESO} \mathrm{EFOSC2}$ & 2000/Mar./30 & 320 & $U B V R I$ & \\
\hline $\mathrm{F}$ & $"$ & $"$ & 1800 & $B$ & TRP; exposures of $20 \mathrm{~s}$ each \\
\hline $\mathrm{G}$ & $3.6 \mathrm{~m} \mathrm{TNG} \mathrm{DoLoReS}$ & 2001/Jan./01 & 21600 & $B V R$ & TRP; exposures of $20 \mathrm{~s}$ each \\
\hline $\mathrm{H}$ & $8.2 \mathrm{~m} \mathrm{VLT-UT1}$ FORS1 & 2001/Jan./16-23 & 18000 & $3600-6000$ & $6 \AA$ res.; grism $600 \mathrm{~B}$; slit $1^{\prime \prime}$ \\
\hline I & $"$ & 2001/Jan./19-24 & 10800 & $6000-9000$ & $30 \AA$ res.; grism $150 \mathrm{I}$; slit $1^{\prime \prime}$ \\
\hline
\end{tabular}

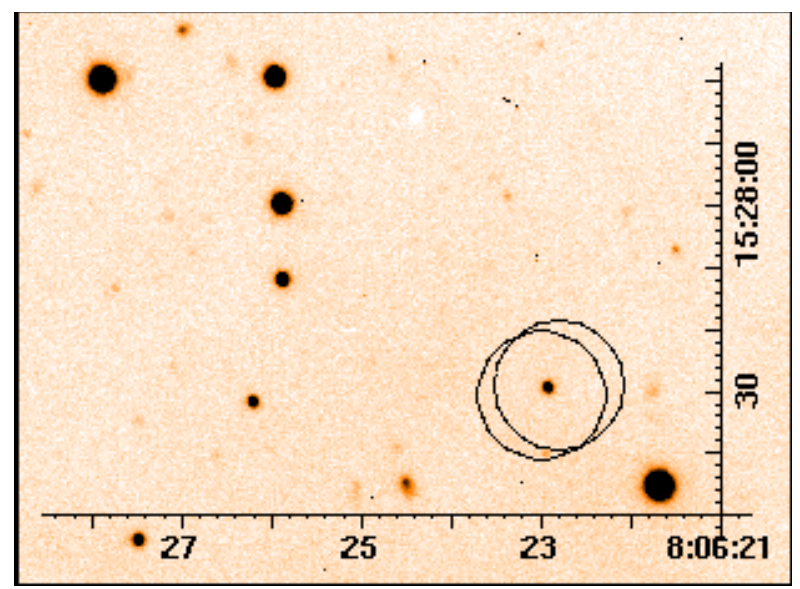

Fig. 1. VLT FORS2 $B$ filter image of the field of RX J0806.3+1527. The circles represents the X-ray uncertainties inferred from the ROSAT HRI observations. Coordinates units are right ascension and declination.

$B$ filter image of the field of RX J0806.3+1527 together with the ROSAT HRI positional uncertainties. Down to the limiting $R$ magnitude of $\sim 25.3$ only one object was found consistent with the HRI error circles. This was at $\mathrm{RA}=08^{\mathrm{h}} 06^{\mathrm{m}} 22^{\mathrm{s}} .9$ and Dec $=+15^{\circ} 27^{\prime} 31^{\prime \prime} .0$ (uncertainty of $\sim 0^{\prime \prime}$.5; equinox 2000), fully consistent with the previously identified blue object (I99). The $U, B, V, R$ and $I$ magnitudes were determined to be 19.6, 20.7, 21.1, 21.0 and 20.9 , respectively (magnitude uncertainties of $\sim 0.1$ ) on March 1999.

Time-resolved photometry (TRP) in the $B$ band was first obtained with the ESO $3.6 \mathrm{~m}$ telescope equipped with EFOSC2 (obs. F) over an interval of $1800 \mathrm{~s}$. Pulsations at the $321 \mathrm{~s} \mathrm{X}$-ray period were detected, with a pulsed fraction (semiamplitude of modulation divided by the mean source magnitude) of $15 \pm 4 \%$ (90\% confidence level) confirming the correctness of the optical counterpart identification.

Based on this result, we observed again the source in the $B, V$ and $R$ bands with the $3.6 \mathrm{~m}$ Telescopio Nazionale Galileo (TNG) equipped with DoLoReS (obs. G). TRP at a time resolution of $20 \mathrm{~s}$ was obtained in each of the three filters $(\sim 2 \mathrm{hr}$ per filter $)$ for a total duration of $7 \mathrm{hr}$. Differential light curves were accumulated by subtracting the source magnitudes with the mean magnitude of 10 reference stars within the field of view. Figure 2 (upper panel) shows the whole light curve of RX J0806.3+1527 normalised, as an example, to one of the reference stars. A best period of $321.5 \pm 0.3 \mathrm{~s}$ was obtained by fitting the phases of the modulation obtained over 6 different intervals of $\sim 4000 \mathrm{~s}$ exposure each. This value is fully consistent with that detected in $\mathrm{X}$-rays $(321.25 \pm 0.25 \mathrm{~s})$, to within $\sim 1$ part in $10^{3}$. The shape of the $B, V$ and $R$ modulations could be well fit by the sum of two sinusoids (fundamental plus 2nd harmonic; see Fig. 2). Pulsed fractions of $13.9 \pm 0.5 \%, 14.2 \pm 0.6 \%$ and $13.2 \pm 0.6 \%$ (90\% confidence level) were determined for the $B, V$ and $R$ band, respectively. In order to search for additional flux modulations up to periods of hours, we merged the $B, V$ and $R$ light curves, by normalising their average flux to the average $B$ band flux and calculated a power spectrum (see Fig. 2); no significant signal was detected (99\% confidence level upper limit of $1.5 \%$ ) in the $1 \mathrm{~min}-5 \mathrm{hr}$ period range, other than that at $321 \mathrm{~s}$. Aperiodic flickering is apparent in the optical light curve.

Several ESO Very Large Telescope (VLT) medium resolution $(6 \AA)$ spectra were obtained with $30 \mathrm{~min}$ exposures in the blue band with FORS1+2 (see Rupprecht \& Böhnhardt 2000 for instrument description; obs. B and H). Each spectrum was analysed independently. The summed and normalised spectrum obtained during obs. $\mathrm{H}$ is shown in Fig. 3 (bias subtracted, flat-field corrected and calibrated in wavelength). No significant absorption features were detected, while several faint (equivalent width, $E W$, of $\sim-1 \div-6 \AA$ ) and broad (full width half maximum, FWHM, of $\sim 20-30 \AA$ ) emission lines are apparent. The lines at $5411 \AA, 4541 \AA, 4199 \AA, 4025 \AA$ and $3923 \AA$ are unambiguously identified with HeII Pickering lines. The lines at $6560 \AA, 4859 \AA, 4338 \AA, 4100 \AA$ and $3968 \AA$ correspond to the even terms of this series. NIII/CIII emission lines around $4640 \AA$ and $5270 \AA$ are also detected testifying that recombination processes are occurring in the system. Intensity changes (up to a factor of $\sim 2$ in 

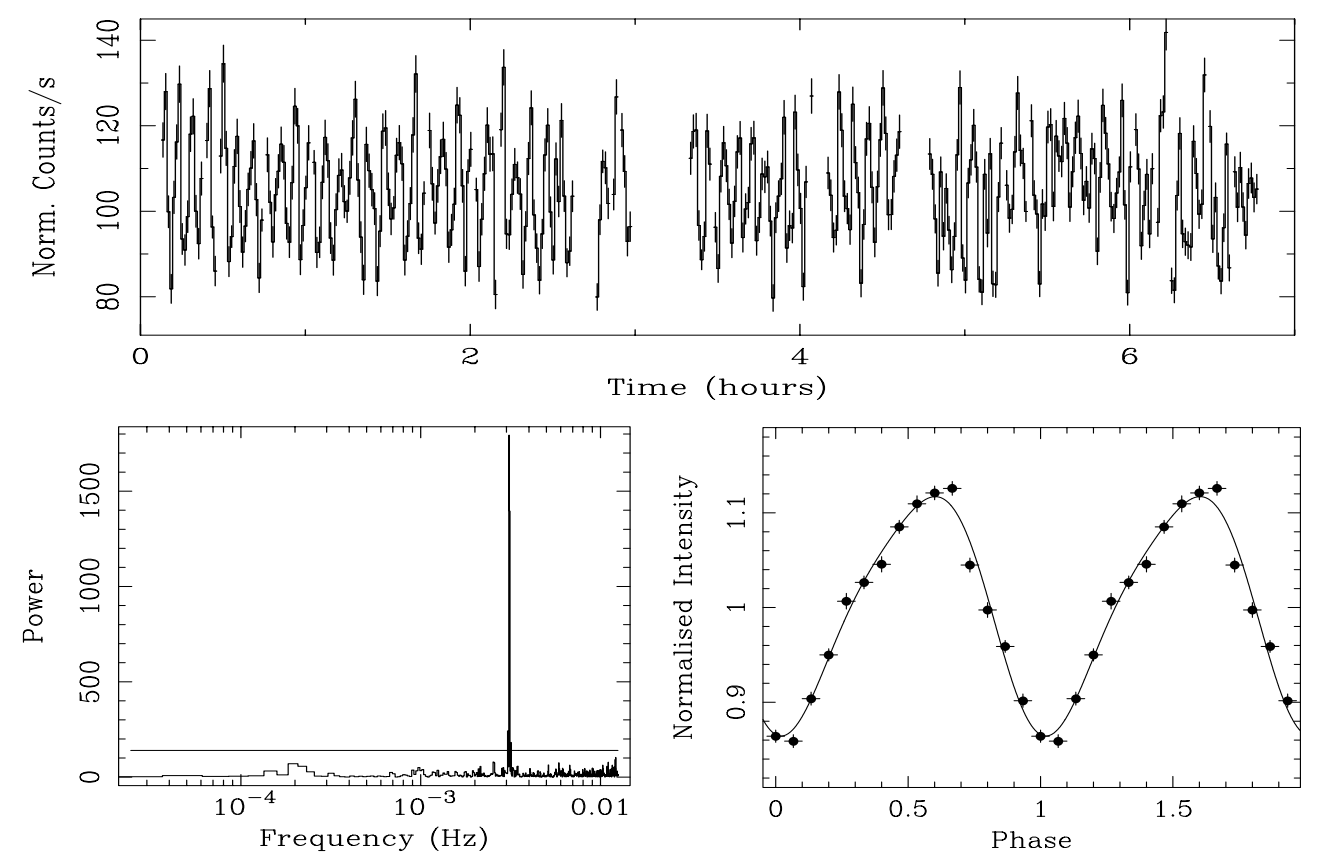

Fig. 2. TNG DoLoReS B (from start to $2.1 \mathrm{hr}), V(2.1-4.5 \mathrm{hr}$ ) and $R$ (4.5 hr to the end) merged light curve for the optical counterpart of RX J0806.3+1527 (upper panel). Power spectrum density with superimposed the $99 \%$ confidence level threshold for signals (lower left panel). Merged $B, V$ and $R$ light curve folded to the best period of $321.5 \mathrm{~s}$, with superimposed the best fit (lower right panel).

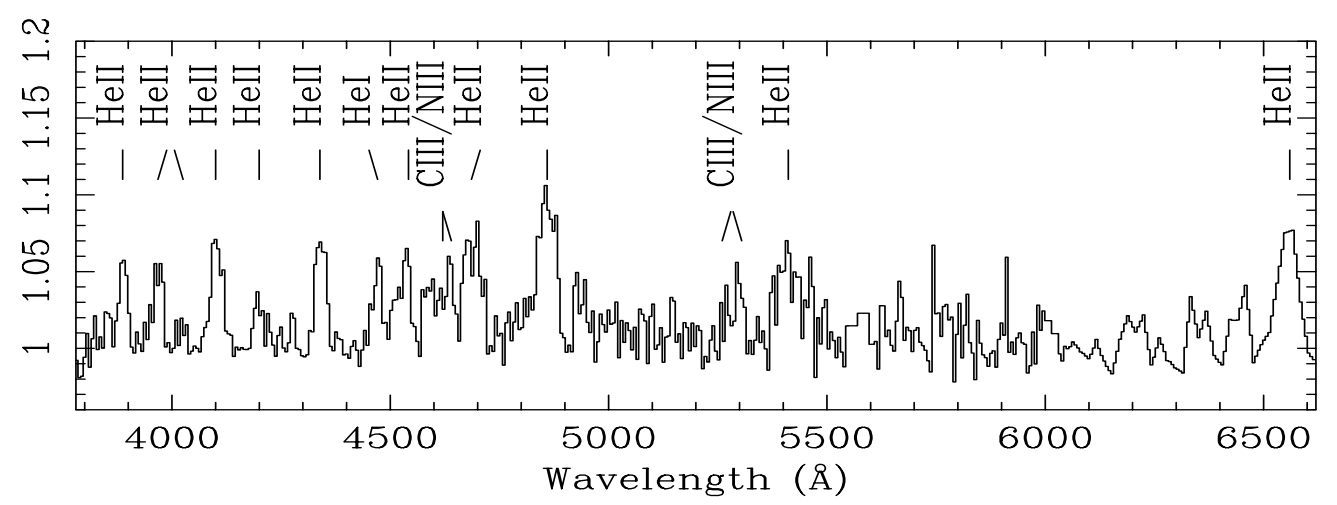

Fig. 3. VLT FORS1 medium $(6 \AA$; $3800-6000 \AA)$ and low $(30 \AA$; above $6000 \AA)$ resolution spectra obtained for the optical counterpart of RX J0806.3+1527. Numerous faint emission lines of HeI and HeII (blended with H) are labeled.

EW) and shape of the emission lines were detected from night to night and even within the same night. VLT low resolution $(30 \AA)$ spectra were also obtained to sample the red part of the spectrum (obs. D and I; exposure times of $15-30 \mathrm{~min}$ ). The low and medium resolution spectra collected in 2001 were flux-calibrated using the spectrum of the $V=13.56 \mathrm{sdB}$ spectrophotometric standard GD108. The calibrated flux is dominated by a steep blue continuum with no intrinsic absorption lines (see Fig. 4). The shape of the optical continuum is thermal and implies a temperature of $T_{\mathrm{bb}}>4 \times 10^{4} \mathrm{~K}$ together with negligible reddening of $E(B-V) \leq 0.01$. Its normalisation is such that its blackbody emitting radius is $R_{\mathrm{bb}} \sim 570(d / 100 \mathrm{pc})\left(T_{\mathrm{bb}} / 4 \times 10^{4} \mathrm{~K}\right)^{-1 / 2} \mathrm{~km}$, with $d$ the source distance.

Spectral information at higher photon energies was obtained by analysing the Extreme Ultraviolet Explorer
(EUVE) survey and the RASS data. The EUVE upper limits were extrapolated from data taken from on-line a strophysical databases. The relevant archival RASS data were retrieved and photon arrival times extracted within a $2^{\prime}$ radius region centered on the peak of the emission. Photons were also extracted from a nearby region, far from other detected sources, so as to have a similar background level. The source ROSAT PSPC Pulse Hight Analyser (PHA) rates were grouped so as to contain a minimum of 10 photons per energy bin (after background subtraction), resulting in 4 statistically independent energy bins. A blackbody model gave a good fit (by using a maximum likelihood and $\mathrm{C}$ statistics) with a characteristic temperature of $T_{\mathrm{bb}} \sim\left(6 \pm_{4}^{13}\right) \times 10^{5} \mathrm{~K}$ (90\% confidence level) and an absorption column of $1.9 \times$ $10^{20} \mathrm{~cm}^{-2}$ (however the $90 \%$ uncertainty includes all values $<6 \times 10^{20} \mathrm{~cm}^{-2}$ ) and a $0.1-2.0 \mathrm{keV}$ unabsorbed 


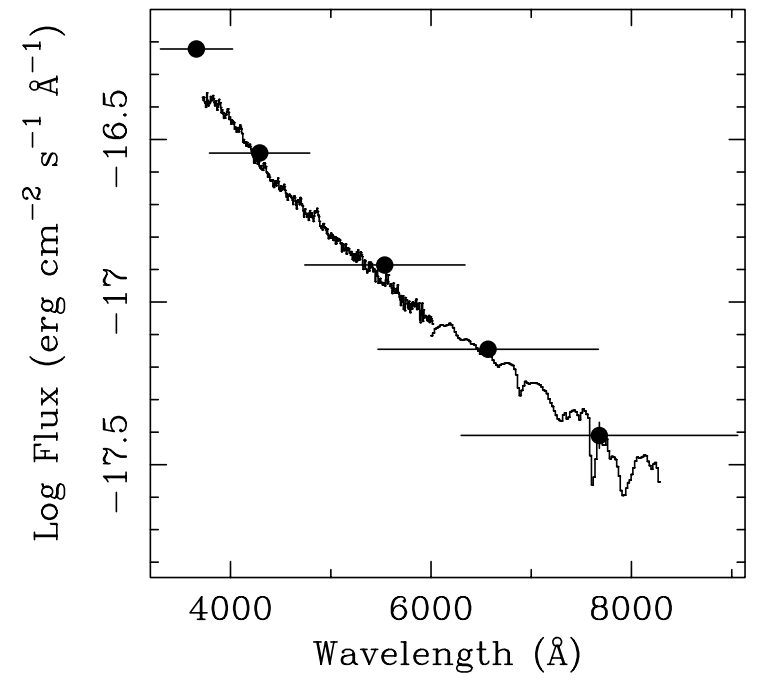

Fig. 4. VLT FORS1 flux-calibrated medium and low resolution spectra with superimposed, from the left to the right, the $U, B, V, R$ and $I$ photometric data.

flux of $\sim 4 \times 10^{-10} \mathrm{erg} \mathrm{cm}^{-2} \mathrm{~s}^{-1}$. We also inferred an unabsorbed flux of $\sim 5 \times 10^{-9} \mathrm{erg} \mathrm{cm}^{-2} \mathrm{~s}^{-1}$ at the peak of the emission of the $321 \mathrm{~s}$ modulation. By combining the $\mathrm{X}$-ray data point and EUVE upper limits with the optical continuum from the VLT observations, we obtained a best fit blackbody spectrum for a temperature of $T \sim 2.6 \times 10^{5} \mathrm{~K}$. This value, however should be treated with caution as it depends crucially on the assumption that the spectrum of RX J0806.3+1527 from the optical to the $\mathrm{X}$-rays is due to a single blackbody emission component. We note that the vastly different amplitude of the $321 \mathrm{~s}$ modulation in the optical and the $\mathrm{X}$-rays argues against this possibility.

Finally, we used the 1951 and 1991 Palomar Observatory Sky Survey plates, digitized to produce the Guide Star Catalogue-II, in conjunction with the ESO frames to put an upper limit of $0.02^{\prime \prime} \mathrm{yr}^{-1}$ on the proper motion of this object. Assuming that we are seeing only the reflex peculiar motion of the sun, this would put the object at a distance of $\geq 100 \mathrm{pc}$.

\section{Discussion}

The results of the optical campaigns reported in this letter led to the unambiguous identification of the optical counterpart of RX J0806.3+1527, and provide also important new constraints on the source.

Firstly, the possibility that RX J0806.3+1527 is a nearby isolated neutron star accreting from the interstellar medium can be ruled out, because for a distance of $\geq 100 \mathrm{pc}$ (as implied by the upper limit on the proper motion) the blackbody emission radius inferred from the optical continuum (or the optical continuum plus the RASS point) would be several hundred $\mathrm{km}$ at least, too large a value for any neutron star model.

Two additional results are especially relevant for assessing the nature of RX J0806.3+1527; these are: (a) the absence of optical periodicities other than the $321 \mathrm{~s}$ modulation (up to periods of $\sim 5 \mathrm{hr}$ ); (b) the He emission line spectra. The absence of a second periodicity argues against models involving a non-synchronous magnetic accreting white dwarf (such as the intermediate polars, IPs). In addition to their orbital and spin periodicities (and/or their beat), IPs display optical spectra with intense Balmer emission lines with $\mathrm{EW}$ of tens of $\AA$ and widths of a few hundred $\mathrm{kms}^{-1}$. Moreover the $\mathrm{X}$-ray amplitude of the spin modulation is comparatively low (Hellier 1999). The $100 \% \mathrm{X}$-ray modulation and broad and weak emission lines that we observed from RX J0806.3+1527 are also very much at variance with these IP properties. Moreover a hot blue thermal continuum similar to the one we revealed from RX J0806.3+1527 is simply not observed in magnetic cataclysmic variables of any class. We note that if the blue thermal continuum were attributed to the accreting white dwarf (and/or the accretion stream) the optical spectra exclude the presence of a companion star earlier than a M7V for a (maximum) distance of $1 \mathrm{kpc}$. The condition that such a (hypothetical) companion star fills its Roche lobe translates into an orbital period of $<2 \mathrm{hr}$, well within the range of periods sampled by our photometric studies. Moreover it would be very difficult the explain the observed He emission lines within such a scenario.

The most natural interpretation of our results is that RX J0806.3+1527 is a double degenerate binary system, in which mass is transferred from a Roche lobe filling white dwarf to another more massive white dwarf. Such binaries belong to the AM CVn class and comprise only a few objects, although recently two new members have been proposed (RX J1914+24, period of $9.5 \mathrm{~min}$; Ramsay et al. 2000, and KUV 01584-0939, period of 10 min; Warner \& Woudt 2002). AM CVns are intrinsically blue objects with thermal optical continua, orbital period in the $10-50 \mathrm{~min}$ range (usually detected in the optical band), fairly broad He emission and absorption lines $(F W H M \sim 15-35 \AA$; for a review see Warner 1995). The presence of flickering in the optical light curve testifies that these systems are powered by accretion. AM CVns are expected to originate from normal composition binaries which experienced two phases of mass exchange, exposing the helium cores of the original stars (e.g. Warner 1995). During their evolution, they reach a minimum orbital period of $\sim 4$ min and thereafter evolve to longer periods. Double-peaked emission line spectra clearly testify to the presence of an accretion disk mediating the flow of matter. However in the X-ray bright AM CVn candidate RX J1914+24, the accretion disk is likely not present (Marsh \& Steeghs 2002; Wu et al. 2002). In one model for such a short orbital period system the soft $\mathrm{X}$-ray emission originates from direct impact accretion which occur when the minimum distance of the gas stream from the center of mass of the accretor is smaller than the accretor size (Marsh \& Steeghs 2002).

We propose here that RX J0806.3+1527 is a new member of the AM CVn class and in particular that: (i) The modulation at $321 \mathrm{~s}$ corresponds to the orbital period of the system. The $100 \%$ amplitude in the $\mathrm{X}$-ray modulation 
can be easily explained in terms of self-occultation of the stream impact point on the surface of the accreting white dwarf. The blue optical continuum and the small amplitude optical modulation likely results from $\mathrm{X}$-ray reprocessing by a fraction of the donor star surface and/or the accretion stream. The similarities with RX J1914+24 suggest that RX J0806.3+1527 might also be a direct impact accretor. In this case the system may be non-synchronous, while no conspicuous modulation is produced at the accreting white dwarf spin period. The possibility that the system is magnetically locked, as in AM Her binaries, cannot be excluded at present, although the observed line EWs and shape of the optical continuum argue against this. (ii) Emission lines from $\mathrm{He}$ are expected given the temperatures implied by the optical continuum and the presence of $\mathrm{H}$ depleted accreting gas. Note that He emission lines were detected also in KUV 01584-0939 (Wegner et al. 1987), whereas the study of the optical spectrum of RX J1914+24 is hampered by the high absorption in the direction of the source (Ramsay et al. 2000, 2002). The presence of flickering in the RX J0806.3+1527 optical light curves (and the X-ray ROSAT HRI light curves as well, see I99) further suggests that the optical emission is at least in part related to the accretion process, as expected in the irradiation scenario.

The condition that the mass donor white dwarf fills its Roche-lobe determines its mass $M_{2}=0.12 M_{\odot}$, and radius $R_{2}=1.7 \times 10^{9} \mathrm{~cm}=0.02 R_{\odot}$. The mass transfer rate $\dot{M}$ driven by angular momentum losses through gravitational radiation can then be calculated over a reasonable accretor mass range. We adopt $0.2 \leq M_{1} / M_{\odot} \leq 0.5$, the lower limit being close to the stable mass transfer limit. This range translates into $1-3 \times 10^{-7} M_{\odot} \mathrm{yr}^{-1}$ (assuming a efficient tidal coupling), an accretion luminosity in the $2-5 \times 10^{35} \mathrm{erg} \mathrm{s}^{-1}$ range and a flux of $10^{-9}-4 \times$ $10^{-7} \mathrm{erg} \mathrm{s}^{-1} \mathrm{~cm}^{-2}$ (for a distance in the $0.1-1 \mathrm{kpc}$ range). The latter values are consistent with the peak flux inferred from the RASS (see Sect. 1). In order not to violate the (minimum) size of the blackbody emitting area derived from the normalisation of the optical continuum $\left(200 \leq R_{\mathrm{bb}}(\mathrm{km}) \leq 6000\right.$ for a distance of $0.1-1 \mathrm{kpc}$ and the entire range of allowed blackbody temperatures) the optical continuum must come from a smaller region than the donor star, perhaps the accretion stream and/or the illuminated part of the donor star.

Within this interpretation the secondary's orbital velocity is expected to be in the $900-1500 \mathrm{~km} \mathrm{~s}^{-1}$ range, such that the observed emission line width might be dominated by the binary's Doppler velocity amplitude. Phaseresolved spectroscopy can ascertain this point unambiguously and provide important new information.
In summary our results provide compelling evidence that RX J0806.3+1527 is a double white dwarf interacting binary system, with the shortest orbital period ever recorded. The source represents one of the most promising targets for gravitational wave detection from binary motion (Nelemans et al. 2001). Indeed a $321 \mathrm{~s}$ orbital period falls well above $\log f \sim-2.7 \mathrm{~Hz}$, where the average galactic background hampers the gravitational wave detection. Moreover assuming reasonable values of the distance and mass of the accretor, a strain amplitude of up to few $\times 10^{-21}$ is expected, which is well above the LISA sensitivity.

Acknowledgements. Based on observations made with the Italian Telescopio Nazionale Galileo (TNG) operated on the island of La Palma by the Centro Galileo Galilei of the CNAA (Consorzio Nazionale per l'Astronomia e l'Astrofisica) at the Spanish Observatorio del Roque de los Muchachos of the Instituto de Astrofisica de Canarias We thank the ESO director for the DDT allocated to observe RX J0806.3+1527 and R. M. Athreya for the help in carrying out observations at the $3.6 \mathrm{~m}$ ESO. We thank G. Tessicini and G. Marino for the observations at the TNG. The Guide Star Catalogue-II is joint project of the Space Telescope Science Institute and the Osservatorio Astronomico di Torino. An anonymous referee provided useful comments on the first version of this paper. This work is supported through CNAA, ASI and MURST grants.

\section{References}

Beuermann, K., Thomas, H.-C., Reinsch, K., et al. 1999, A\&A, 347,47

Burwitz, V., \& Reinsch, K. 2001, AIP Conf. Proc., 599, 522

Hellier, C. 1999, in the Annapolis Workshop on Magnetic Cataclysmic Variables, ASP Ser., 157

Israel, G. L., Panzera, M. R., Campana, S., et al. 1999, A\&A, 349, L1 (I99)

Marsh, T. R., \& Steeghs, D. 2002, MNRAS, in press [astro-ph/0201309]

Nelemans, G., Yungelson, L. R., \& Portegies Zwart, S. F. 2001, A\&A, 375, 890

Ramsay, G., Cropper, M., Wu, K., Mason, K. O. \& Hakala, P. 2000, MNRAS, 311, 75

Ramsay, G., Wu, K., Cropper, M., et al. 2002, MNRAS, in press [astro-ph/0202281]

Rupprecht, G., Böhnhardt, H. (eds.) 2000, FORS1+2 User Manual V1.4, VLT-MAN-ESO-13100-1543

Warner, B. 1995, Ap\&SS, 225, 249

Warner, B., \& Woudt, P. A. 2002, PASP, 114, 129

Wegner, G., McMahan, K., \& Boley, F. I. 1987, AJ, 94, 1271

Wu, K., Cropper, M., Ramsay, G, \& Sekiguchi, K. 2002, MNRAS, in press [astro-ph/0111358] 